\title{
A NOTE ON REDUCTIVE OPERATORS
}

\author{
FRANK GILFEATHER ${ }^{1}$
}

\begin{abstract}
A bounded linear operator $A$ on a Hilbert space is called reductive if every invariant subspace of $\boldsymbol{A}$ reduces it. This paper gives examples of operators which give an affirmative answer to the reductive question: If $A$ is reductive, then is $A$ normal?
\end{abstract}

The reductive question is significant since its affirmative answer for all bounded operators is an equivalent formulation of the well-known invariant subspace question [6]. Since the latter question remains unsettled in general, several attempts have been made to determine for which classes of operators the answer to the reductive question is affirmative. At present, there are essentially three types of operators which have been shown to give an affirmative answer to the reductive question. The first type involves compactness, another involves scalar type operators in the sense of N. Dunford, and the thrid involves combinations of the first two types.

The purpose of this note is to consider these results and show that they (and generalizations) can be obtained from certain structure theorems of the author. The results involving compactness originally stem from the affirmative answer to the reduction question given by T. Ando [1] and based on the Aronszajn and Smith invariant subspace theorem [2]. We shall show that Ando's result, as well as generalizations of results combining compactness and scalar operator arguments, will follow from a structure theorem for an operator $T$ for which a noncommutative polynomial $p\left(T, T^{*}\right)$ is compact [7]. We note that some form of a known invariant subspace theorem will also be used in this approach.

A generalization of the result that a scalar type reductive operator is normal is that a spectral operator of finite type (that is, nilpotent quasi-nilpotent part) if reductive is normal. This easy generalization does lead to a new class of operators for which the reductive question is affirmative. Namely, we show that a $\operatorname{root} A$ of a locally nonzero abelian

Received by the editors May 17, 1973 and, in revised form, August 31, 1973.

AMS (MOS) subject classifications (1970). Primary 47A15, 47B15; Secondary 47B05, 47B40.

Key words and phrases. Reductive operator, invariant subspace.

${ }^{1}$ This research was supported by NSF Grant GP-31435. 
analytic function $\psi$ if reductive is normal; cf. [8]. From this, it follows that if there exists an analytic function $f$ defined on a domain containing $\sigma(A)$, the spectrum of an operator $A$, and not constant on any open set intersecting $\sigma(A)$, and if $A$ is reductive while $f(A)$ is normal, then $A$ is normal. Thus, in particular, the only reductive $n$th roots of a normal operator are normal.

Let $T$ be a spectral type operator and let $T=S+Q$ be its canonical decomposition into scalar type operator $S$ and quasi-nilpotent operator $Q$; cf. [5]. Whenever $Q$ is compact, there exists a family of mutually disjoint invariant subspaces $\left\{H_{i}\right\}$ for $T$, so that $H$ is the algebraic direct sum of the subspaces $H=H_{0} \dot{+} H_{1} \dot{+} \cdots$, and correspondingly $T=T_{0} \dot{+}$ $T_{1}+\cdots$ where $T_{i}$ is $T$ restricted to $H_{i}$. Moreover, $T_{0}$ is a scalar type operator and each $T_{i}$ is of the form $\lambda_{i} I_{i}+Q_{i}$ where $Q_{i}$ is $Q$ restricted to $H_{i}$ and $Q_{i}$ is irreducible (Theorem 4 of [7]; cf. also [3]). From this, we can easily obtain the following result announced in [10].

THEOREM 1. A spectral operator $T$ with compact quasi-nilpotent part if reductive is normal.

Proof. Since $T$ is reductive, the subspaces $H_{i}$ mentioned above each reduce $T$. For $i \geqq 1$ the operator $Q_{i}$ is compact, irreducible, and also reductive. Hence any invariant subspace of $Q_{i}$ would reduce it, and thus $H_{i}$ must be one dimensional whenever $i \geqq 1$. It is easy to see that a reductive scalar type spectral operator is normal and thus $T_{0}$ is also normal (cf. Remark 2 below). Since each $H_{i}$ reduces $T$, and $T$ restricted to each of these subspaces is normal, it follows that $T$ is normal.

REMARK 1. Using Lemma 2 in [7] it follows that a reductive spectral type operator $T$ is normal if any of the following operators (i) $T^{*} T-T T^{*}$, (ii) $T^{*}-T$, (iii) $I-T^{*} T$, or (iv) $T T^{*} T-T^{*} T^{2}$ is compact.

REMARK 2. Let $S$ be a reductive scalar type spectral operator with spectral measure $E(\cdot)$. If $E=E(\delta)$ is a spectral projection for $S$, then since $S$ is reductive, $E$ commutes with the orthogonal projection $P$ on $E H$. Then $(I-E) E=(I-E) P=0$ as well as $E P=E=E^{2}$. Therefore $E-P=E(E-P)+(I-E)(E-P)=0$ and the spectral measure $E(\cdot)$ is selfadjoint. Thus we have shown $S$ is a normal operator (this result was announced in [9] and [10]).

REMARK 3. Let $T$ be of the form $N+K$, where $N$ is normal and $K$ is a compact operator commuting with $N$. The eigenspaces of $K$ are easily seen to be invariant for $N$ and, by the Fuglede theorem, also for $N^{*}$. Thus, if $T$ is reductive, $T$ restricted to an eigenspace of $K$ is normal so that the reductive question for such a $T$ reduces to the question for spectral operators with compact quasi-nilpotent part. Thus, for such $T$ the answer to the reductive question is affirmative. This was first announced 
in [9] and [10] where $N$ and $K$ commute, leaving the much harder question where $N$ and $K$ do not commute still unsolved.

A second use of the results in [7] yields the following decomposition for an operator $T$ for which there is a noncommutative polynomial $p(z, \bar{z})$ such that $p\left(T, T^{*}\right)$ is compact. Here we may decompose $H$ by mutually orthogonal reducing subspaces $H_{i}$ of $T$ so that $H=H_{0} \oplus H_{1} \oplus \cdots$, and the decomposition of $T$ is $T=T_{0} \oplus T_{1} \oplus \cdots$ where $p\left(T_{0}, T_{0}^{*}\right)=0$ and $T_{i}(i \geqq 1)$ is irreducible with $p\left(T_{i}, T_{i}^{*}\right)$ also compact (Theorem 2, [3], [7]). From this, it follows that the reductive question is affirmative whenever $T$ is polynomially compact [6], [12].

Whenever a translate of an operator $T$ times a translate of $T^{*}$ is a compact operator, then it follows that $T$ has a nontrivial invariant subspace, and we can show that if such an operator $T$ is reductive it must be normal.

PROPOSITION 1. Let $T$ satisfy $T^{*} T+\alpha T+\beta T^{*}+\gamma I$ is compact where $\alpha \neq \bar{\beta}$ or $|\alpha|^{2}=\gamma$, then $T$ has a nontrivial invariant subspace. Moreover, if $T$ is reductive, then $T$ is normal.

Proof. If we let $\hat{A}$ be the image of $A$ in the Calkin algebra, then $\hat{T}^{*} \hat{T}+\alpha \hat{T}+\beta \hat{T}^{*}+\gamma \hat{I}=0$. This expression factors as $\left(\hat{T}^{*}+\alpha \hat{I}\right)(\hat{T}+\beta \hat{I})=c \hat{I}$ where $\alpha \beta-\gamma=c$. Let $T_{1}=T+\beta I$, then $\left(\hat{T}_{1}^{*}+a \hat{I}\right) \hat{T}_{1}=c \hat{I}$ and assume $a=\alpha-\bar{\beta} \neq 0$. Thus,

and

$$
c \hat{I}-a \hat{T}_{1}=\hat{T}_{1}^{*} \hat{T}_{1}=\bar{c} \hat{I}-\bar{a} T_{1}^{*},
$$

$$
\hat{T}_{1}^{*}=\bar{a}^{-1}(\bar{c}-c) \hat{I}+\bar{a}^{-1} a \hat{T}_{1} .
$$

Since $\hat{T}_{1}^{*} \hat{T}_{1}+a \hat{T}_{1}-c \hat{I}=0$, it follows that

$$
\bar{a}^{-1} a \hat{T}_{1}^{2}+\left(a+\bar{a}^{-1}(\bar{c}-c)\right) \hat{T}_{1}-c \hat{I}=0
$$

or $T$ is polynomially compact. Since $\bar{a}^{-1} a \neq 0$, it follows that $p(T)$ is compact for a monic polynomial $p$, and therefore, $T$ has a nontrivial invariant subspace [4]. Finally, by using the remarks which precede the proposition, if $T$ is reductive, then it is normal.

In what follows, let $\mathscr{C}$ denote the closed ideal of compact operators on $H$, $\mathscr{P}(T)$ the weakly closed algebra generated by $T$ and $I$, and $\mathscr{C}_{T}=\mathscr{P}(T) \cap \mathscr{C}$. A theorem of Lomonosov which appears as a corollary in [11] implies that if $\mathscr{C}_{T} \neq 0$, then $T$ has a nontrivial invariant subspace. Denote by $\left[T, T^{*}, \mathscr{C}_{T} H\right]$ the span of vectors formed by applying polynomials in $T$ and $T^{*}$ to vectors in the range of $C H$ for some $C \in \mathscr{C}_{T}$.

Proposition 2. Let $T$ be an operator with $\left[T, T^{*}, \mathscr{C}_{T} H\right]=H$. If $T$ is reductive, then it is normal. 
Proof. This proposition follows by using Proposition 1 in [7] and an analogue of Theorem 3 in [7] as well as the recent Lomonosov invariant subspace theorem.

A locally nonzero abelian analytic function $\psi$ is an operator valued analytic function which takes values in an abelian von Neumann algebra $\mathscr{A}$, and for which there does not exist a sequence $\left\{z_{n}\right\} \rightarrow z_{0}$ in its domain of definition for which $\bigcap \mathscr{N}\left(\psi\left(z_{n}\right)\right) \neq 0(\mathscr{N}(A)$ is the null space of $A)$. An operator $T$ is called a root of the abelian analytic function $\psi$ if $T \in \mathscr{A}^{\prime}$ (the commutant of $\mathscr{A}$ ), $\sigma(T) \subset \mathscr{D}$ (the domain of definition for $\psi$ ) and

$$
0=\psi(T) \equiv(2 \pi i)^{-1} \int_{\Gamma}(T-z I)^{-1} \psi(z) d z
$$

where $\Gamma$ is an admissible curve in $\mathscr{D}$ containing $\sigma(T)$ in its interior [8].

THEOREM 2. If $T$ is a root of a locally nonzero abelian analytic function and $T$ is reductive, then $T$ is normal.

PROOF. The structure of roots of a locally nonzero abelian analytic function has been given in [8]. Such operators are piecewise spectral operators of finite type. Specifically, there exist projections $\left\{P_{n}\right\}$ with $I=\sum P_{n}, P_{n} P_{m}=\delta_{n m} P_{m}$ so that $P_{n} T=T P_{n}$, and $T / P_{n} H$ is a spectral operator of finite type. It is easy to see that a reductive operator which is a spectral operator of finite type is normal [10]. Consequently, $T$ is the direct sum of normal operators; hence it is normal.

A scalar valued analytic function is called locally nonconstant if its derivative does not identically vanish on some subdomain of its domain of definition. Let $T$ be an operator with $\sigma(T)$ contained in the domain of a locally nonconstant analytic function $f$. If $f(T)=N$ where $N$ is a normal operator and $\psi(z)=f(z) I-N$, then $\psi$ is a locally nonzero abelian analytic function, and $T$ is one of its roots [8].

COROLlaRY. Let $f$ be a locally nonconstant analytic function with domain $\mathscr{D}$. If $T$ is a reductive operator with $\sigma(T) \subset \mathscr{D}$ and if $f(T)$ is a normal operator, then $T$ is a normal operator.

Of course, polynomials are locally nonconstant so this result states that if $T$ is reductive and $p(T)$ is normal for some polynomial $p$, then $T$ is normal.

An operator $A$ is called $n$-normal if it is an $n \times n$ matrix of commuting normal operators on a direct sum of Hilbert spaces. Since $n$-normal operators satisfy a locally nonzero abelian analytic function, we may also conclude that if reductive they are normal. This result was independently discovered by C. K. Fong of the University of Toronto. 


\section{REFERENCES}

1. T. Ando, A note on invariant subspaces of a compact normal operator, Arch. Math. 14 (1963), 337-340. MR 27 \#4073.

2. N. Aronszajn and K. T. Smith, Invariant subspaces of completely continuous operators, Ann. of Math, (2) 60 (1954), 345-350. MR 16, 488.

3. H. Behncke, Structure of certain nonnormal operators. II, Indiana Univ. Math. J. 22 (1972), 301-308.

4. A. R. Bernstein and A. Robinson, Solution of an invariant subspace problem of K. T. Smith and P. R. Halmos, Pacific J. Math. 16 (1966), 421-431. MR 33 \#1724.

5. N. Dunford and J. T. Schwartz, Linear operators. III, Wiley, Interscience, New York, 1971.

6. J. A. Dyer, E. A. Pedersen and P. Porcelli, An equivalent formulation of the invariant subspace conjecture, Bull. Amer. Math. Soc. 78 (1972), 1020-1024.

7. F. Gilfeather, On the Suzuki structure theory for non self-adjoint operators on Hilbert space, Acta Sci. Math. (Szeged) 32 (1971), 239-249.

8. - Operator valued roots of abelian analytic functions, Pacific J. Math. (to appear).

9. R. L. Moore, Properties of reductive operators, Notices Amer. Math. Soc. 20 (1973), A159.

10. E. A. Nordgren, H. Radjavi and P. Rosenthal, Compact perturbations of normal operators with reducing invariant subspaces, Notices Amer. Math. Soc. 20 (1973), A155; and Address \#509, American Mathematical Society 79th Annual Meeting, January 26, 1973.

11. C. Pearcy and A. Shields, A survey of some recent results on invariant subspaces of operators on Banach spaces, Mimeographed notes, 1973.

12. P. Rosenthal, Completely reducible operators, Proc. Amer. Math. Soc. 19 (1968), 826-830.

Department of Mathematics, University of Nebraska, Lincoln, Nebraska 68508 